\title{
Study on formation mechanism of YSZ thermal barrier coatings with segmented structure
}

\author{
Yi Wang ${ }^{1, a^{*}}$, Shuo Sun ${ }^{2, b}$, Min Liu ${ }^{3, c}$ and Hongli Suo ${ }^{4, d}$ \\ ${ }^{1}$ School of Materials Science and Engineering \\ Beijing University of Technology \\ 100124, Beijing, China \\ a wangyibg@bjut.edu.cn
}

\begin{abstract}
Keywords: Thermal barrier coatings (TBCs), Segmentation cracks, Formation mechanism, Tensile strength

Abstract. Compared with traditional structures, YSZ TBCs with high segmentation crack density sprayed have exhibited a promising potential in improving TBC thermal shock resistance because the segmentation cracks increase its strain tolerance. A study is presented of formation mechanism of segmented TBC. The segmentation cracks initiate and propagate during the deposition phase, as a result of stress relaxation when the coating is subjected to tensile stress. Also, the crack density reaches a constant value and is not influenced by further plastic deformation of the metal. High heat input to the substrate is the most favorable factor in developing segmentation cracks because particle temperature promote the joining of adjacent lamellae.
\end{abstract}

\section{Introduction}

Thermal barrier coatings have been widely used for protection of hot-section components of aircrafts. Typical TBCs with a two-layer structure, consisting of the metallic bond coat and $6-8 \% \mathrm{Y}_{2} \mathrm{O}_{3}$ stabilized zirconia (YSZ) top coat ${ }^{[1,2]}$, can be prepared by two different processes: plasma spraying (PS) and electron beam-physical vapor deposition (EB-PVD) techniques. Compared with the traditional structure, which is obtained through the process of plasma spraying, YSZ-TBCs under a "hot condition"exhibit a higher segmentation crack density, which could significantly upgrade the strain tolerance of the TBCs ${ }^{[3-5]}$. The results indicate that the YSZ-TBCs have the distinct potential to improve the thermal shock resistance of TBCs.

The objective of the present work is to investigate the influence of deposition conditions on the structures of atmospheric plasma-sprayed thermal barrier coatings. Further, an analysis of the formation of segmentation cracks of the TBCs will be performed.

\section{Experimental}

\section{Coating preparation}

Ni-25Cr-6Al-1Y powders (Institute of Metal Research, China) and $\mathrm{ZrO} 2-8$ mass- $\% \mathrm{Y}_{2} \mathrm{O}_{3}$ powder (Sulzer Metco 204NS) were used as spraying materials for the MCrAlY bond coat and the YSZ topcoat respectively. The YSZ TBCs were produced by PS with Sulzer Metco PS units. High velocity oxy-fuel was used to deposit an $80-120 \mathrm{~mm}$ NiCrAlY bond coat onto a disc shaped Ni-based super-alloy substrates with diameters of $20 \mathrm{~mm}$ and thicknesses of $3 \mathrm{~mm}$. The coatings of YSZ were atmospheric plasma sprayed onto the NiCrAlY-coated super-alloy substrates. Segmentation cracks were caused by thermal tensile stress during the deposition process. The substrate should have a heat input that is high enough to obtain strong bonds between lamellae, which is necessary for proper propagation. Following these ideas, a short spray distance, a high plasma power, and a preheated substrate were used. The parameters used for spraying YSZ TBCs are listed in Table 1. 
Table 1 Processing parameters for spraying YSZ coatings

\begin{tabular}{|c|c|c|c|c|c|}
\hline$g$ Coatin & $\begin{array}{l}\text { Power } \\
(\mathrm{KW})\end{array}$ & $\begin{array}{c}\text { Distance } \\
(\mathrm{mm})\end{array}$ & $\begin{array}{l}A r(\text { slpm }) / \\
H_{2} \text { (slpm) }\end{array}$ & $\begin{array}{l}\text { Feed rate } \\
(\mathrm{g} / \mathrm{min})\end{array}$ & $\begin{array}{c}\text { Gun } \\
\text { Velocity } \\
(\mathrm{mm} / \mathrm{s})\end{array}$ \\
\hline $\mathrm{A}$ & 40 & 70 & $45 / 10$ & 30 & 500 \\
\hline B & 45 & 60 & $45 / 12$ & 30 & 500 \\
\hline
\end{tabular}

\section{Results and discussion}

\section{Microstructure and phase of sprayed YSZ coatings}

Fig.1(a) and Fig.1(b) show SEM images of cross-sections of the sprayed YSZ coatings with spray sets A and B, as listed in Table.1 respectively. Coating A reveals a typical lamellar structure, with a YSZ coating that is approximately $200 \mathrm{~mm}$ thick. In contrast to coating A, coating B contains several segmentation cracks. The crack density was measured to be $3.8 \mathrm{~mm}-1$. In addition, there were some branching cracks which are cracks parallel to the coating plane.

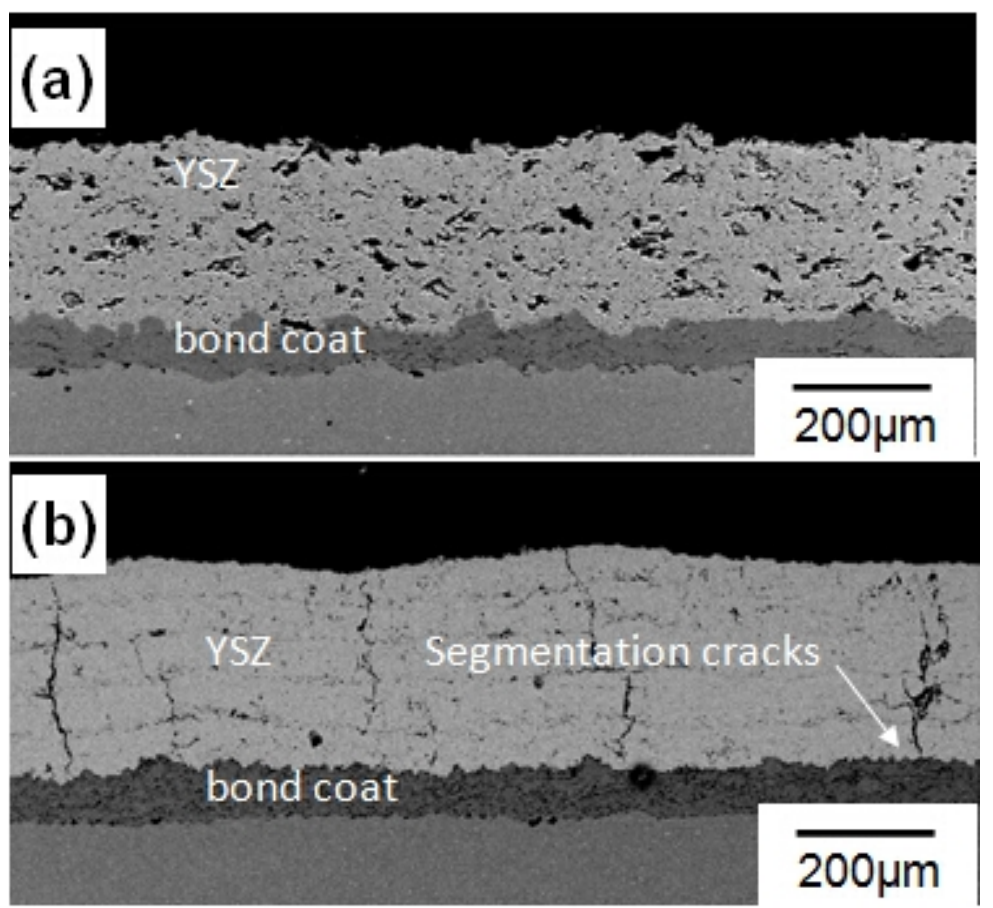

Fig.1 Shows SEM of the sprayed YSZ coating a) YSZ coatings; b) YSZ with segmentation cracks.

\section{Development of segmentation cracks}

The stress resulting from the contraction of individual splats is specific to the spraying process. The origin of the stress is constrained by the underlying solid body on the thermal contraction of sprayed splats, as shown schematically in Fig. 2. As a molten particle strikes the surface of a solid, it typically spreads out, forming a flat splat and rapidly losing heat to the underlying solid through conduction. A good joining between lamellae is a key for the propagation of segmentation cracks ${ }^{[6-7]}$. Accordingly, a high heat input to the substrate is one of the most important factors in developing segmentation cracks because high substrate and particle temperature promote the joining of adjacent lamellae. 

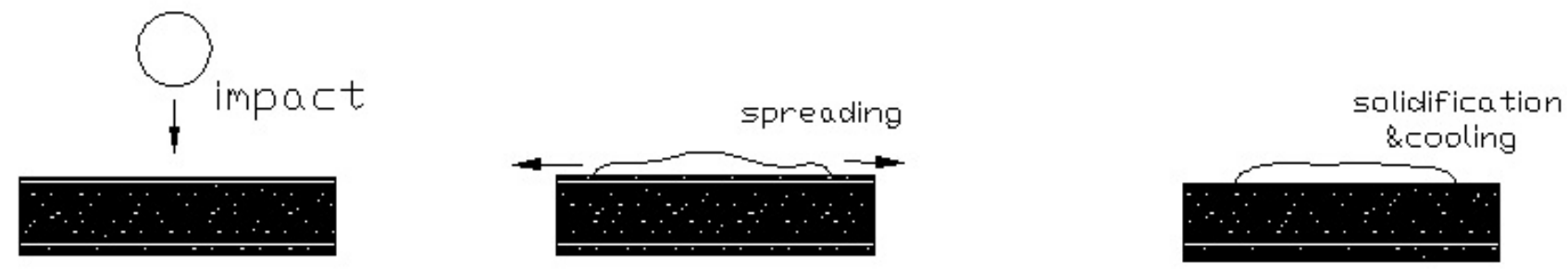

Fig.2 Schematic depiction of impact, spreading and cooling of a single splat.

A model is used to explain the generation of segmentation cracks ${ }^{[8]}$. Assuming that film thickness hc in the model is much smaller than substrate thickness hs, it can be said that:

ec=es $\quad \operatorname{Eq}(1)$

where ec and es are the coating and the substrate strain, respectively. As the applied tension increases, both the substrate strain and the film strain increase. When the in-plane stress $\sigma \mathrm{C}$ in the film reaches the film strength $\sigma_{\mathrm{c}}{ }^{*}$, a crack will be introduced into the film as shown in Fig. 3(a). Then, the in-plane stress is relaxed due the free surface effect near the crack. If one considers the force balance of the left half of one segment (hatched region), the results are shown in Eq. (2).

$$
\int_{0}^{\lambda / 2} \tau \mathrm{dx}=\int_{0}^{h c} \sigma c \mathrm{dz}
$$

where $\lambda$ is the length of the segment. As the substrate is strained further, the film can be more finely segmented. However, it was shown that beyond a certain es, the crack density $1 / \lambda$ saturates, as shown in Fig. 3(b). This was replicated by experiments, as well, and can be understood using Eq. (3). Eq. (3) allows us to see that the maximum value of $\sigma_{\mathrm{c}}$ is a constant $\sigma_{\mathrm{c}}{ }^{*}$ and the integral on the right-hand side should reach approximately a constant value if the substrate is further strained to the point just before

$$
\begin{gathered}
\sigma_{\mathrm{c}} \text { reaches } \sigma_{\mathrm{c}}{ }^{*} . \\
\tau^{*}=\pi h c \frac{\sigma c^{*}}{\lambda *}
\end{gathered}
$$

On the other hand, as the segment size $\lambda$ decreases, $\tau$ needs to increase in order to maintain force balance. In the referenced analysis, modified sinusoidal shear stress distributions were assumed for $\tau$. As $\lambda$ becomes small, $\tau$ starts to reach its maximum possible value $\tau^{*} \square$ (the interfacial shear strength) before $\tau$ reaches $\tau^{*}$. After this happens, segmentation cracks no longer develop, and the interface between the film and the substrate will be destroyed as the substrate strain increases further, as show in Fig. 3(c). When the spraying was performed at a high substrate temperature, the temperature between two adjacent splats could be high enough to lead to a partial remelting of the surface of underlying splats. Thus, the tensile stresses generated in the recently deposited splats will be relaxed by the propagation of microcracks ${ }^{[7]}$. Following this procedure, the microcracks will finally go through the coating, developing into segmentation cracks, as shown in Fig. 3(d). On the contrary, at a low substrate temperature, the contact area between splats is very limited. As a result, a large amount of interlamellar 
gaps, or horizontal cracks, will exist in the coating. These cracks or gaps will lead to a significant stress relaxation. In such case, the microcracks in the individual splats could not propagate into segmentation cracks.
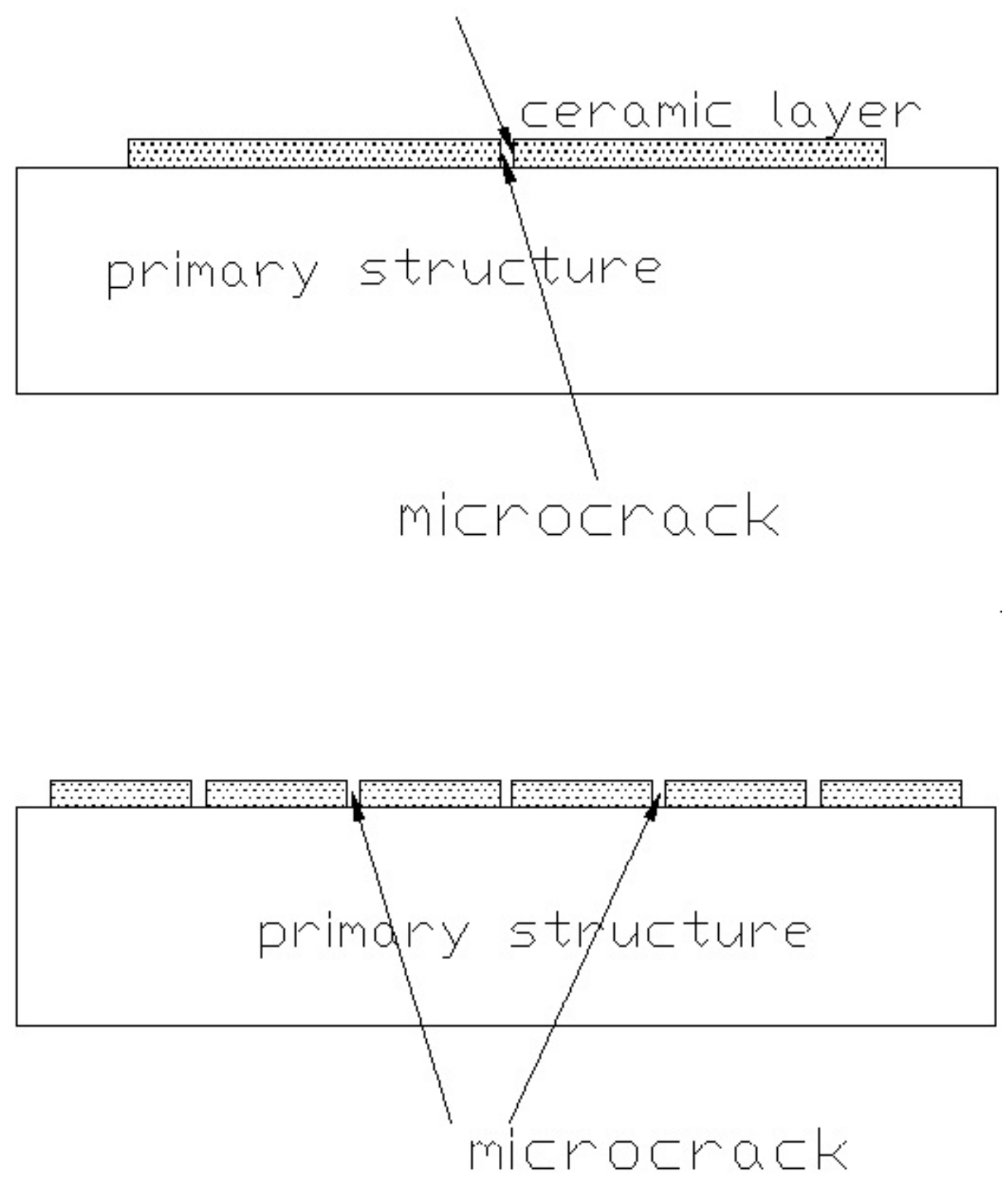

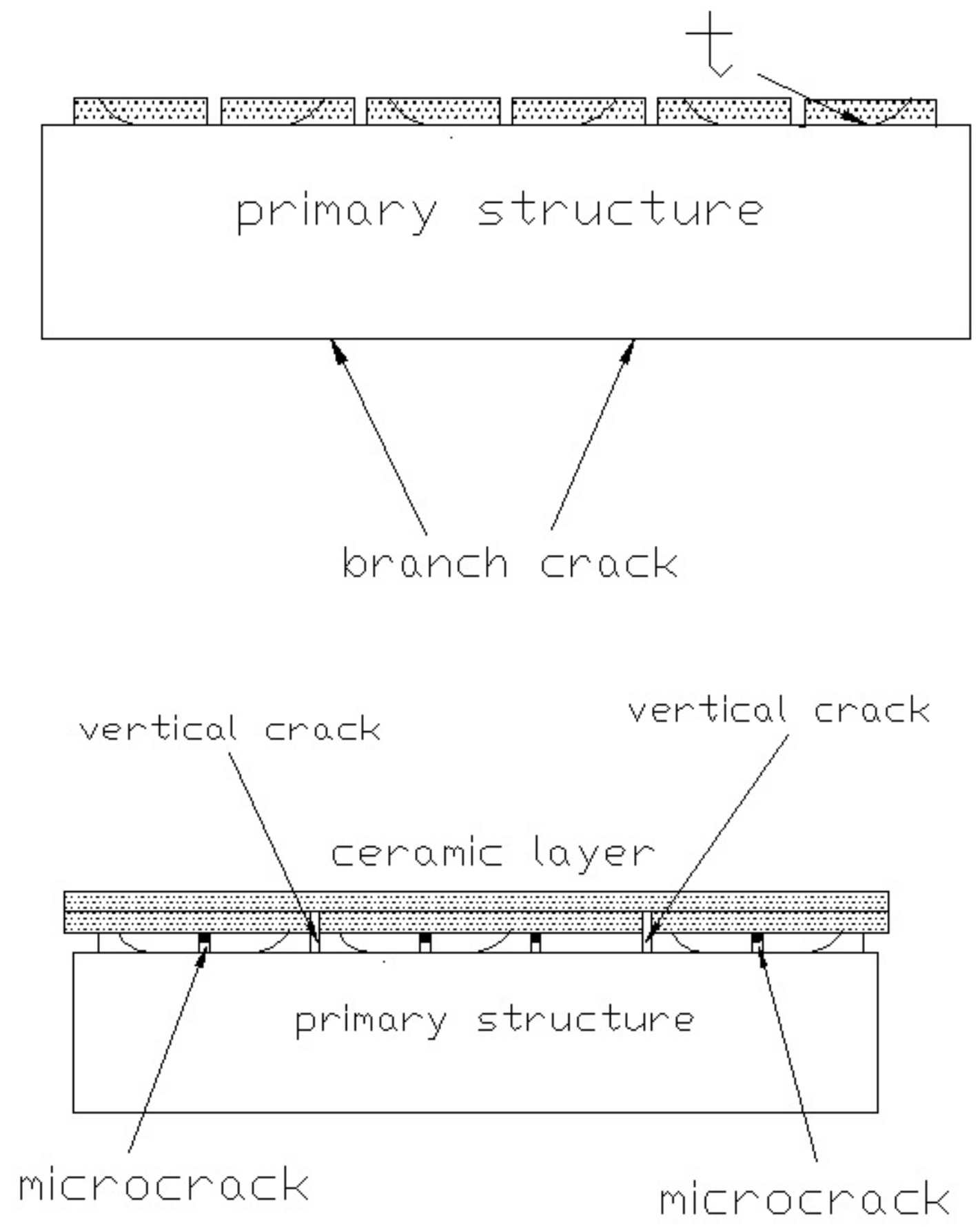

Fig. 3 Model explaining the generation of segmentation cracks.

Substrate temperature has an important impact on structure features of the sprayed coatings. It is established that a high substrate temperature can give rise to a high segmentation crack density. The segmentation cracks increase the strain tolerance of TBC. The observed branching cracks between layers as shown in Fig. 4 are a sign that the differential strain of one pass was large enough to cause interfacial cracking. The branching cracks, which run perpendicular to the direction of heat flux, can reduce the thermal conductivity of $\mathrm{TBC}^{[8]}$. 


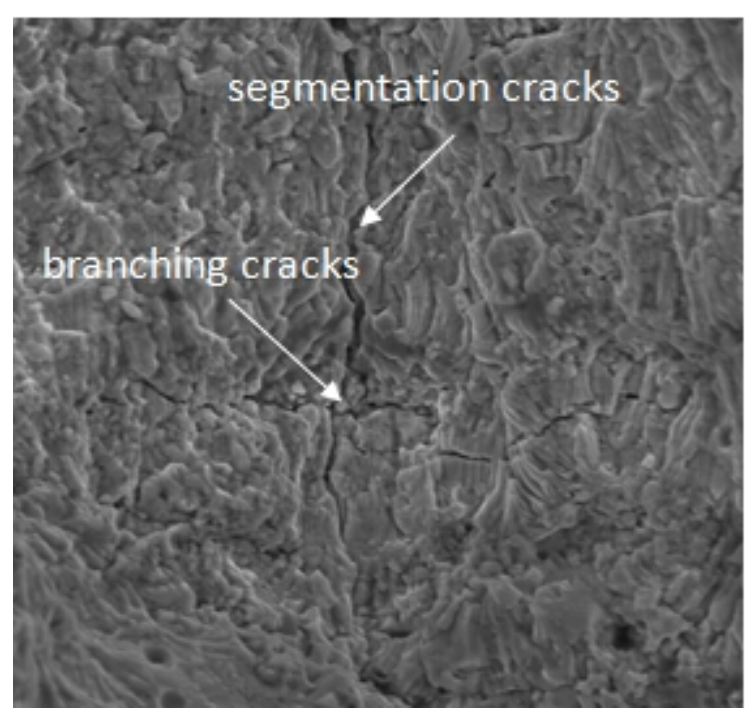

Fig.4 Segmentation cracks and associated branching cracks.

\section{Conclusions}

The temperature of the substrate is important to the development of segmentation cracks. When spraying was performed with a high substrate temperature, the temperature between the first splat and the following splat will be high enough to lead to strong bonds between splats partially by remelting of the surface of the first splat. The tensile stresses developed in the second splat will be relaxed by the propagation of microcracks preexisting in the first splat. Following this procedure, the microcracks will finally go through the coating, developing into segmentation cracks.

\section{Acknowledgement}

The authors gratefully acknowledge the financial support of China National Funds for Distinguished Young Scientists (No.51401003).

\section{References}

[1] K. Bobzin, N. Bagcivan and T. Brögelmann: 'Influence of temperature on phase stability and thermal conductivity of single- and double-ceramic-layer EB-PVD TBC top coats consisting of 7YSZ, Gd2Zr2O7 and La2Zr2O7', Surf. Coat. Technol., 2013, 237, 56-64.

[2] Y. Wang, M. Li and H. Suo: 'Mechanical properties of YSZ thermal barrier coatings with segmented structure', Surface engineering ., 2012, 28, 329-332.

[3] Y. Wang, H. Guo and Z. Li: 'Segmented Lanthanum Cerium Oxide Thermal Barrier Coatings By Atmospheric Plasma Spray', Surface Engineer., 2009, 25, 555-558.

[4] S. Zhao, Y. Zhao and B. L. Zou: 'Characterization and thermal cycling behavior of La2(Zr0.7Ce0.3)2O7/8YSZ functionally graded thermal barrier coating prepared by atmospheric plasma spraying', Journal of Alloys and Compounds., 2014, 592, 109-114.

[5] J. Xiang, S. Chert and J. Huang: 'Synthesis kinetics and thermophysieal properties of La2(Zr0.7Ce0.3)207 ceramic for thermal barrier coatings', Journal of Rare Earths., 2012, 30, 228-232.

[6] H. Dong, D. Wang and Y. L. Pei: 'Optimization and thermal cycling behavior of La2Ce2O7 thermal barrier coatings', Ceramics International., 2013, 39, 1863-1870. 
[7] X. Chen, Y. Zhang and X. Zhong: 'Thermal cycling behaviors of the plasma sprayed thermal barrier coatings of hexaluminates with magnetoplumbite structure', Journal of the European Ceramic Society., 2010, 30, 1649-1657.

[8] Y. Wang, H. Guo and S. Gong: 'Thermal Shock Resistance and Mechanical Properties of La2Ce2O7 Thermal Barrier Coatings with Segmented Structure', Ceramics International., 2009, 35, 2639-2644. 